\title{
Hormones, médiateurs et glomérules
}

Le glomérule rénal ne peut plus être considéré seulement comme un filtre passif. La filtration glomérulaire est soumise à une régulation hormonale, en particulier par l'angiotensine II et les prostaglandines. En outre, les cellules glomérulaires synthétisent de nombreux médiateurs.

\section{Raymond Ardaillou Professeur de physiologie}

\section{ADRESSE}

R. Ardaillou : Inserm 64, Hôpital Tenon, 4, rue de la Chine, 75020 Paris. es paramètres déterminant l'ultrafiltration glomérulaire ont été mesurés directement par microponction, pour la première fois en I97I, en utilisant une souche mutante de rats (Wistar-Munich) aux glomérules superficiels [I]. Le débit de filtration glomérulaire (DFG) dépend de plusieurs facteurs : la différence des pressions hydrostatique et oncotique s'exerçant de part et d'autre de la paroi des capillaires glomérulaires, le flux plasmatique glomérulaire, la surface de filtration disponible et la perméabilité à l'eau de la paroi du capillaire. En conséquence, la régulation peut opérer à différents niveaux, soit à l'extérieur du glomérule par modification des résistances artériolaires pré- et post glomérulaires, soit à l'intérieur du glomérule luimême par changement de la surface de filtration disponible ou de la perméabilité à l'eau de la paroi du capillaire. Dans les dix dernières années, de nombreuses études menées essentiellement dans les laboratoires de Brenner et de Blantz ont apporté des informations sur la nature des hormones et des médiateurs qui modifient le DFG et sur leur mécanisme d'action $[2,3]$. Ce type de travail réalisé in vivo ne peut établir ni le site d'action à l'intérieur du glomérule, ni le mode d'action intracellulaire de la substance .étudiée. De plus, les expériences de microponction glomérulaire sont limitées au rat et évidemment inutilisables chez l'homme. C'est pourquoi nous avons appliqué les techniques de biochimie et d'endocrinologie cellulaire à l'étude du glomérule isolé et des cellules glomérulaires en culture.

\section{Structures glomérulaires étudiées}

Dans la plupart des travaux, des glomérules isolés d'origine murine ou humaine ont été utilisés. Ces glomérules sont préparés par des procédés purement mécaniques. Les préparations obtenues sont pures à $95 \%$. Elles contiennent des touffes de capillaires glomérulaires sans artérioles et sans capsule de Bowman. Comme le glomérule est un organe complexe composé d'au moins trois lignées cellulaires et d'une membrane basale, ce genre de préparation ne permet pas de rattacher une propriété biochimique à un type cellulaire déterminé. Aussi, des cultures de lignées homogènes de cellules glomérulaires ont-elles été également étudiées. Deux types cellulaires ont pu être cultivés à partir des glomérules humains et murins, les cellules mésangiales et les cellules épithéliales. Ces cellules sont identifiées par leurs caractères morphologiques en microscopie 
électronique, par la présence dans les cellules mésangiales et l'absence dans les cellules épithéliales de faisceaux de filaments de myosine révélés par immunofluorescence avec des anticorps antimyosine, par la contractilité des cellules mésangiales en présence d'angiotensine II (AII) et enfin par leur capacité de synthèse de différents types de collagène et glycosaminoglycans. Les cellules épithéliales et mésangiales humaines sont clonées et étudiées après plusieurs passages. Celles de rat peuvent également être clonées ou étudiées en culture primaire. En effet, les cellules épithéliales se multiplient rapidement à la périphérie des glomérules et sont obtenues en lignée homogène au $6^{\mathrm{e}}$ jour. Elles disparaissent ensuite, puis les cellules mésangiales se multiplient et sont obtenues en lignée homogène au $21^{\mathrm{eme}}$ jour de la culture primaire.

\section{Récepteurs glomérulaires}

L'existence de récepteurs hormonaux dans les glomérules a été démontrée soit directement en utilisant les techniques classiques de liaison de l'hormone marquée, soit indirectement par l'effet de l'hormone sur une activité biologique, effet qui implique l'existence de récepteurs. En fait, les deux types d'études sont inséparables; on ne peut en effet affirmer l'existence de récepteurs spécifiques, que s'ils sont liés à une activité biologique. Le tableau I indique quels récepteurs spécifiques ont été trouvés sur les glomérules et les cellules glomérulaires.

L'hormone la plus étudiée a été l'AII parce qu'elle joue un rôle essentiel dans le maintien des résis-

\begin{tabular}{|c|c|c|c|c|}
\hline \multicolumn{5}{|c|}{$\begin{array}{c}\text { Tableau I } \\
\text { RÉCEPTEURS GLOMÉRULAIRES MURINS ET HUMAINS }\end{array}$} \\
\hline agoniste & technique d'étude & localisation & $\begin{array}{l}\text { Ko ou ED50 } \\
\text { (rat) }\end{array}$ & $\begin{array}{l}\text { Ko ou EDso } \\
\text { (homme) }\end{array}$ \\
\hline All & liaison & $\begin{array}{l}\text { glomérules } \\
\text { cellules mésangiales }\end{array}$ & $\begin{array}{l}50-100 \mathrm{pM} \\
3 \mathrm{nM}\end{array}$ & $0,1-2 \mathrm{nM}$ \\
\hline AVP & liaison & cellules mésangiales & $10 \mathrm{nM}$ & \\
\hline PTH & $\begin{array}{l}\text { liaison } \\
\text { activation de l'adenylate cyclase } \\
\text { stimulation de l'AMPc endogène }\end{array}$ & $\begin{array}{l}\text { glomérules } \\
\text { glomérules } \\
\text { glomérules }\end{array}$ & $\begin{array}{l}0,1 \mu \mathrm{M} \\
0,1 \mu \mathrm{M} \\
0,25 \mu \mathrm{M}\end{array}$ & $10-110 \mathrm{nM}$ \\
\hline ANF & $\begin{array}{l}\text { stimulation de la guanylate } \\
\text { cyclase }\end{array}$ & glomérules & & $0,25 \mu \mathrm{M}$ \\
\hline histamine & $\begin{array}{l}\text { stimulation de l'AMPc endogène } \\
\text { liaison de la cimétidine (récep- } \\
\text { teurs } H \text { 2) }\end{array}$ & $\begin{array}{l}\text { glomérules } \\
\text { glomérules }\end{array}$ & $\begin{array}{l}7 \mu \mathrm{M} \\
3 \mu \mathrm{M}\end{array}$ & \\
\hline insuline & liaison & glomérules & $20 \mathrm{nM}$ & \\
\hline sérotonine & stimulation de I'AMPc endogène & glomérules & $0,5 \mu \mathrm{M}$ & \\
\hline dopamine & $\begin{array}{l}\text { liaison de l'halopéridol (récep- } \\
\text { teurs D 2) }\end{array}$ & glomérules & $5 \mathrm{nM}$ & \\
\hline $\mathrm{PGE}_{2}$ & $\begin{array}{l}\text { liaison } \\
\text { activation de l'adénylate cyclase } \\
\text { stimulation de l'AMPc endogène }\end{array}$ & $\begin{array}{l}\text { glomérules } \\
\text { glomérules } \\
\text { glomérules } \\
\text { cellules mésangiales et épithé- } \\
\text { liales }\end{array}$ & $\begin{array}{r}80 \mathrm{nM} \\
200 \mathrm{nM} \\
5-20 \mu \mathrm{M}\end{array}$ & $0,4-1,6 \mu \mathrm{M}$ \\
\hline $\mathrm{PG} \mathbf{I}_{2}$ & $\begin{array}{l}\text { stimulation de l'AMPc endogène } \\
\text { activation de l'adenylate cyclase }\end{array}$ & $\begin{array}{l}\text { glomérules } \\
\text { cellules mésangiales et épithé- } \\
\text { liales } \\
\text { glomérules }\end{array}$ & $0,2-5 \mu \mathrm{M}$ & $90-460 \mathrm{nM}$ \\
\hline $\mathrm{LTC}_{4}$ & liaison & cellules épithéliales & & $220 \mathrm{nM}$ \\
\hline
\end{tabular}

ANF : facteur natriurétique auriculaire; $K_{0}$ : constante de dissociation apparente; ED50 : dose efficace à $50 \%$; $L T$ : leucotriène; AVP : arginine-vasopressine; PTH : parathormone; All : angiotensine II; PG : prostaglandine. 
tances vasculaires rénales et la régulation de la filtration glomérulaire. A des doses modérément hypertensives, l'administration d'AII à des rats aboutit à la réduction du $\mathrm{Kf}$ (produit de la surface de filtration par le coefficient de perméabilité à l'eau) et du flux plasmatique glomérulaire et, simultanément, à l'élévation de la résistance de l'artériole efférente et de la pression hydrostatique régnant dans les capillaires glomérulaires [4]. Les effets de l'AII sur la microcirculation glomérulaire sont potentialisés par les inhibiteurs de la cyclo-oxygénase [5] et inhibés par les bloqueurs des canaux calciques [6]. L'AII formée dans les reins contribue de manière essentielle à la réduction du Kf observée dans de nombreuses conditions expérimentales : déficit chronique en sodium, dénervation rénale aiguë, obstruction urétérale et plusieurs modèles d'insuffisance rénale aiguë [7]. Les expériences in vitro ont permis d'analyser le mécanisme des effets glomérulaires de l'AII. Celle-ci se lie aux glomérules isolés murins et humains avec tous les caractères d'une liaison spécifique: haute affinité correspondant à la concentration circulante de l'hormone (50 pM), stéréospécificité, réversibilité $[8,9]$. Les récepteurs de l'AII sont essentiellement localisés sur les cellules mésangiales comme cela a été montré en utilisant, soit des cellules cultivées, soit des cellules fraîches préparées à partir de glomérules dissociés. La liaison de l'AII à ses récepteurs spécifiques est modifiée in vitro par les cations divalents, calcium et magnésium, qui l'augmentent et par le guanosine triphosphate qui la diminue [9]. Le nombre et l'affinité des récepteurs de l'AII sont également susceptibles d'être modulés in vivo. C'est ainsi que les glomérules isolés de rats nourris avec un régime riche en sodium fixent plus d'AII, alors que ceux isolés de rats nourris avec un régime pauvre en sodium fixent moins d'AII. Ce résultat semble dû à une régulation du nombre de récepteurs dans le sens de la diminution par l'AII circulante [II]. La modulation in vivo du nombre de récepteurs a été également observée dans des états pathologiques. Par exem- ple, les glomérules de rats rendus diabétiques par la streptozotocyne, comme ceux de rats ayant une glomérulonéphrite par injection d'anticorps antimembrane basale glomérulaire, possèdent moins de récepteurs de l'AII que les glomérules de rats normaux. Ces récepteurs de l'AII sont liés à un double effet biologique dans la cellule : la contraction et la production de prostaglandines (PG). In vitro, l'AII produit une diminution de surface des glomérules isolés et des cellules mésangiales d'environ Io \% à des concentrations identiques à celles nécessaires à la fixation spécifique $[8$, Io]. Comme la fixation, la contraction est stimulée par le calcium, réversible après lavage et inhibée par les antagonistes compétitifs de l'AII. La diminution de surface des cellules mésangiales s'accompagne d'un changement dans la distribution des fibrilles de myosine intracellulaire. Le fait que l'ÁII a un effet évident sur la morphologie des glomérules isolés et des cellules mésangiales in vitro, suggère que cette hormone modifie la surface interne des capillaires glomérulaires in vivo, par la contraction des cellules mésangiales. Le coefficient d'ultrafiltration $\mathrm{Kf}$ étant le produit de la perméabilité hydraulique $k$ et de la surface de filtration S, la chute de ce coefficient après administration d'AII peut donc être attribuée à la diminution de S. Cependant, la pertinence de cette conclusion a été mise en question récemment, car aucune modification du diamètre des capillaires glomérulaires n'a été constatée, après administration d'AII, chez des rats dont la microcirculation rénale était examinée sous microscopie de fluorescence équipée d'un système vidéo de haute sensibilité [12]. Il se pourrait donc que l'AII accroisse plutôt le nombre de capillaires peu perfusés par un changement dans la distribution du flux glomérulaire. Les effets de l'AII sur la contraction des glomérules isolés et des cellules glomérulaires en culture sont inhibés par la $\mathrm{PGE}_{2}$ et l'acide arachidonique alors qu'ils sont stimulés par les inhibiteurs de la cyclo-oxygénase. Ces résultats suggèrent donc que les $P G$ et la $\mathrm{PGE}_{2}$ en particulier, modulent 
les effets de l'AII. Il a été montré d'autre part que l'AII stimule la production de PG par les glomérules isolés et les cellules mésangiales en culture. Des résultats récents obtenus dans une étude utilisant les cellules mésangiales humaines en culture indiquent que l'AII agit en deux minutes et augmente deux à trois fois la production basale de $\mathrm{PGE}_{2}$ et 6-céto-PGF ${ }_{1} \alpha$. Le seuil de stimulation est observé pour une concentration d'AII d'environ Io $\mathrm{pM}$ [13]. Les effets de l'AII sont bloqués par les antagonistes compétitifs de l'hormone et les bloqueurs des canaux calciques.

En résumé, l'AII produit à la fois la contraction des cellules mésangiales et la stimulation dans ces cellules de la synthèse des PG. Plus récemment, il a été également montré que
l'AII augmentait la concentration intracellulaire de calcium et d'inositol I, 4, 5-triphosphate, ce dernier métabolite étant considéré comme l'agent spécifique produisant la translocation du calcium à partir de ses lieux de stockage intracellulaire. Tous ces résultats peuvent être intégrés dans un schéma d'ensemble du mode d'action de l'AII dans les cellules mésangiales glomérulaires (figure I). L'AII se lie à des récepteurs spécifiques situés sur la membrane cellulaire qui sont couplés à la phospholipase C. L'hydrolyse du phosphatidylinositol-4, 5 biphosphate, résultant de l'activité de cette enzyme, est la source de deux messagers, l'inositoltriphosphate et le diacylglycerol. L'inositoltriphosphate augmente la concentration intracellulaire du calcium ionisé. Il en résulte l'activation de la protéine liant le calcium, la calmoduline. Le complexe calciumcalmoduline contrôle l'activité de la kinase de la chaîne légère de la myosine qui assure la phosphorylation de la myosine, permettant ainsi la contraction musculaire. La kinase de la chaîne légère de la myosine est elle-même contrôlée par sa propre phosphorylation qui dépend d'une autre protéine kinase activée par l'AMP cyclique. L'enzyme phosphorylée perd son activité, ce qui aboutit à la relaxation de la cellule. Le diacylglycerol libère de l'acide arachidonique et stimule la protéine kinase C. La $\mathrm{PGE}_{2}$ formée dans les cellules mésangiales à partir de l'acide arachidonique libre, stimule l'adénylate cyclase et accroît le contenu cellulaire en AMP cyclique.

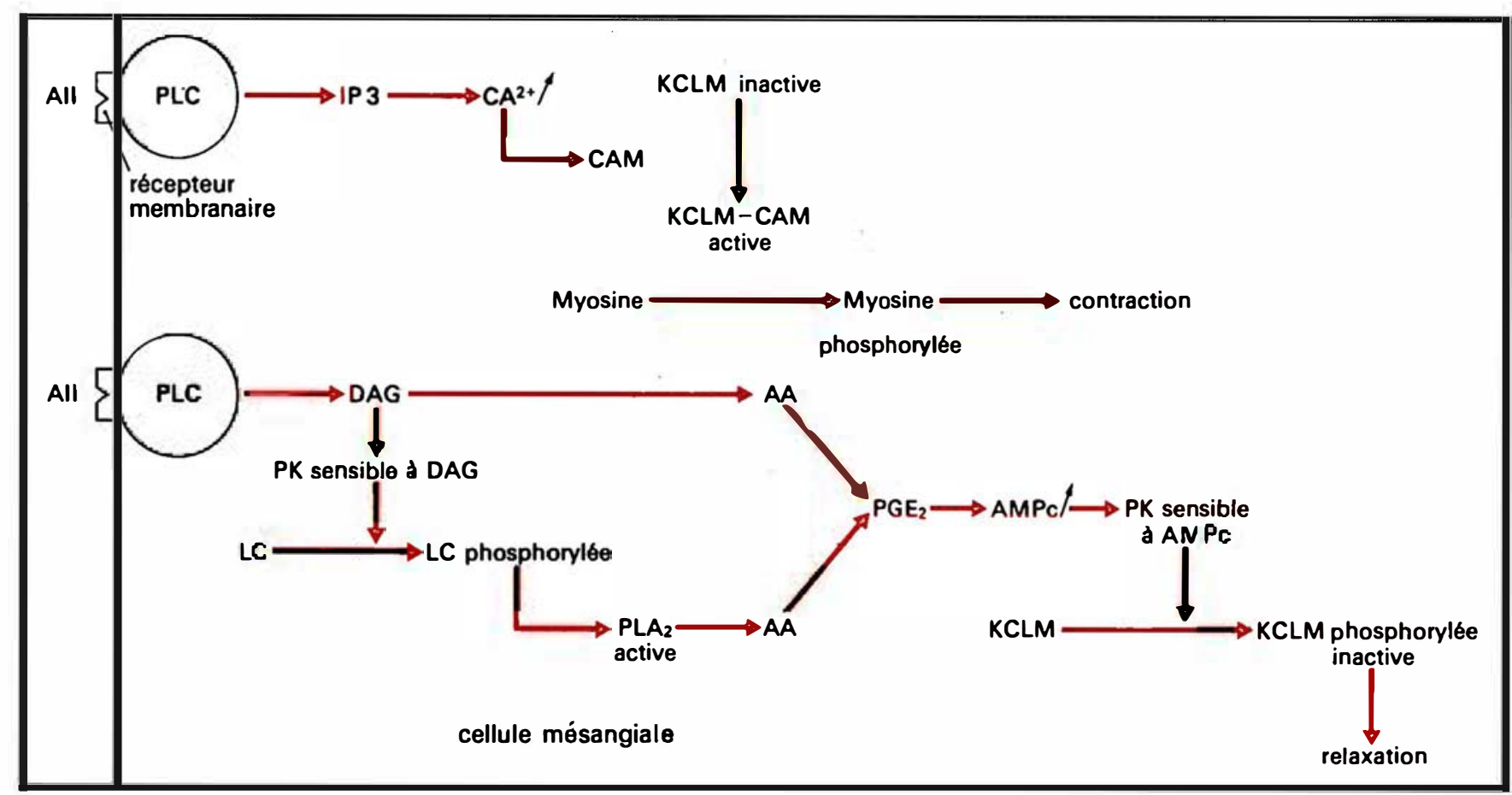

Figure 1. Schéma représentant le mode d'action cellulaire de l'angiotensine |l dans les cellules mésangiales glomérulaires. PLC= phospholipase $C$; IP $3=$ inositoltriphosphate; $K C L M=k i n a s e$ de la chaine légère de la myosine; $D A G=$ diacylglycerol; $P K=$ protéine kinase; $P L A_{2}=$ phospholipase $A_{2} \cdot L C=$ lipocortine; $A M P c=$ adenosine monophos phate cyclique; $P G E_{2}=$ prosta glandine $E_{2} ; A / l=$ angiotensine II; $C A M=$ calmoduline activée par le calcium; $A A=$ acide arachidonique. 


\section{REFERENCES}

14. Ardaillou, N, Nivez MP, Ardaillou R. Stimulation of guanylate cyclase by atrial natriuretic factor in isolated human glomeruli. FEBS Letters $1985 ; 189: 8-11$.

I 5. Baud L, Sraer J, Perez J, Nivez MP, Ardaillou $R$. Leukotriene $C_{4}$ binds to glomerular epithelial cells and promotes their proliferation in vitro. $7 \mathrm{Cl}$ in Invest $1985 ; 76: 374-7$.

16. Sracr J, Ardaillou N, Sracr JD, Ardaillou R. In vitro prostaglandin synthesis by human glomeruli and papillae. Prostaglandins 1982; $23: 855$ 64.

17. Camussi G. Potential role of plateletactivating factor in renal pathophysiology. Kidney Int $1986 ; 29: 469-77$.

18. Sraer J, Rigaud M, Bens M, Rabinovitch $H$, Ardaillou R. Metabolism of arachidonic acid via the lipoxygenase pathway in human and murine glomeruli. 7 Biol Chem 1983; 258 : 4325-3?o.

19. Baud L, Sraer J, Delarue F, et al. Lipoxygenase products mediate the attachment of rat macrophages to glomeruli in vitro. Kidney Int 1985; $27: 855-63$.

20. Lianos EA, Andres GA, Dunn MJ. Glomerular prostaglandin and thromboxane synthesis in rat nephrotoxic serum nephritis. $7 \mathrm{Cl}$ in Invest 1983; 72 : 1439-48.

21. Baud L, Hagege J, Sraer J, Rondeau E, Perez J, Ardaillou R. Reactive oxygen production by cultured rat glomerular mesangial cells during phagocytosis is associated with stimulation of lipoxygenase activity. 7 Exp Med 1983; 158 : 1836-52.

22. Schreiner GF, Kiely JM, Cotran RS, Unanue ER. Characterization of resident glomerular cells in the rat expressing the I a-determinants and manifesting genetically restricted interactions with lymphocytes. $7 \mathrm{Cl}$ in Invest 1981; 68 : 92031 .

23. Camilleri JP, Phat VN, Bariety J, Corvol P, Mènard J. Use of a specific antiserum for renin detection in human kidney. 7 Histochem Cytochem 1980; $28: 1343-6$.

24. Chansel D, Ardaillou N, Ardaillou R. Identification and regulation of renin in human glomeruli and cultured mesangial cells. $\mathrm{Am}$ Soc Nephrol : 18th annual meeting.

25. Kurtz A, Jelkmann W, Pfeilschifter J, Bauer C. Role of prostaglandins in hypoxia-stimulated erytropoictin production. Am $\mathcal{F}$ Physiol 1985; 249 : $C_{3}-8$.
Donc, l'AII produit à la fois la contraction cellulaire et, par l'intermédiaire des $\mathrm{PG}$, le frein à cette contraction cellulaire.

Les glomérules sont également le siège de récepteurs pour d'autres hormones et médiateurs. Plusieurs de ces agents ont en commun leur mode d'action sur l'adénylate cyclase dont ils stimulent l'activité. C'est le cas de l'hormone parathyroidienne (PTH), de l'histamine, de la sérotonine, des $\mathrm{PGE}_{2}$ et $\mathrm{I}_{2}$. L'étude de ces diverses substances in vivo a montré qu'elles réduisaient le $\mathrm{Kf}$ et que cet effet était bloqué par les antagonistes compétitifs de l'AII [2]. De ces résultats, a découlé l'hypothèse suivante : produit sous l'effet de la stimulation de l'adénylate cyclase, l'AMP cyclique stimulerait la production locale de rénine aboutissant à la production d'AII et ainsi à la réduction du $\mathrm{Kf}$. Il n'est pas sûr que ce schéma recouvre toutes les possibilités. La PTH, dont on sait qu'elle est vasodilatatrice par relaxation du muscle lisse, a peut-être une action directe sur les cellules mésangiales. L'effet glomérulaire de deux autres hormones, l'AVP et le facteur natriurétique auriculaire (ANF) ont été également étudiés. L'AVP se lie spécifiquement aux cellules mésangiales et stimule leur contraction [13]. Il s'agit là d'un effet dépendant des récepteurs $V_{\text {I }}$ qui ne sont pas liés à l'adénylate cyclase.

L'ANF se lie également aux glomérules et stimule la guanylate cyclase particulaire [14]. Cet effet est très particulier puisque les agents cholinergiques et le nitroprussiate activent seulement la guanylate cyclase soluble. L'ANF est connu d'autre part comme agent relaxant du muscle lisse vasculaire et a été décrit dans certains cas comme capable d'augmenter le débit de filtration glomérulaire. La relation entre l'augmentation de la concentration de GMP cyclique et ce dernier effet n'est pas encore déterminée. Il se peut que le GMP cyclique diminue la concentration de calcium intracellulaire en favorisant la séquestration et l'efflux de cet ion, ce qui aboutirait à la relaxation des cellules mésangiales.

Les effets glomérulaires des hormones ou médiateurs qui ont été rap- portés jusqu'à présent, sont essentiellement en relation avec la contraction ou la relaxation des cellules mésangiales. Un autre effet a été décrit plus récemment, celui des leucotriènes (LT) $\mathrm{C}_{4}$ et $\mathrm{D}_{4}$ sur la prolifération cellulaire. Le $\mathrm{LTC}_{4}$ se fixe spécifiquement aux cellules glomérulaires épithéliales humaines et provoque parallèlement la multiplication de ces cellules [15]. Il s'agit là d'un fait majeur parce que, au cours de certains types de glomérulonéphrites expérimentales ou humaines, les capillaires glomérulaires sont envahis par des cellules du sang, polynucléaires ou monocytes-macrophages qui synthétisent les LT à partir de l'acide arachidonique par la voie de la 5-lipoxygénase. Les médiateurs produits localement sont donc capables de stimuler la prolifération des cellules épithéliales glomérulaires qui participent à la formation des croissants extracapillaires.

\section{Fonctions de synthèse du glomérule}

Les glomérules isolés et les cellules glomérulaires en culture sont capables de synthétiser de multiples produits dont la liste est indiquée sur le tableau II. La plupart de ces produits, métabolites de l'acide arachidonique, formes actives de l'oxygène, histamine, enzymes protéolytiques, interleukine I sont, à des titres divers, des médiateurs locaux de l'inflammation. D'autres, comme la rénine, jouent un rôle dans le mécanisme de l'équilibre glomérulo-tubulaire. D'autres enfin, comme l'érythropoiétine sont de véritables hormones.

Les médiateurs glomérulaires de l'inflammation qui interviennent dans le mécanisme des lésions glomérulaires au cours des glomérulonéphrites expérimentales ont une double source. Ils sont synthétisés par les cellules sanguines s'accumulant durant ces maladies dans les capillaires glomérulaires, essentiellement les polynucléaires, mais aussi les plaquettes et les monocytes-macrophages. En outre, il a été démontré que les cellules glomérulaires résidantes, principalement les cellules mésangiales, étaient capables elles aussi de les synthétiser. 
Tableau II

FONCTIONS DE SYNTHĖSE DU GLOMÉRULE MURIN ET HUMAIN

\begin{tabular}{|c|c|c|c|c|}
\hline produit & technique d'étude & localisation & espèce & ordre d'abondance \\
\hline PG & $\begin{array}{l}R I+H P L C \\
R I+H P L C \\
R I+H P L C \\
R I+H P L C\end{array}$ & $\begin{array}{l}\text { glomérules } \\
\text { glomérules } \\
\text { cellules épithéliales } \\
\text { et mésangiales } \\
\text { cellules épithéliales } \\
\text { et mésangiales }\end{array}$ & $\begin{array}{l}\text { rat } \\
\text { homme } \\
\text { rat } \\
\text { homme }\end{array}$ & $\begin{array}{l}\mathrm{PGE}_{2}=\mathrm{PGF}_{2_{\alpha}}>\mathrm{TXB}_{2}>\mathrm{PG}_{2} \\
\mathrm{PG}_{2}>\mathrm{TXB}_{2}>\mathrm{PGE}_{2}=\mathrm{PGF}_{2} \\
\mathrm{PGE}_{2}>\mathrm{PGF}_{2} \\
\text { taux de synthèse très bas }\end{array}$ \\
\hline HETE & $\begin{array}{l}\text { HPLC } \\
\text { HPLC }\end{array}$ & $\begin{array}{l}\text { glomérules } \\
\text { glomérules }\end{array}$ & $\begin{array}{l}\text { rat } \\
\text { homme }\end{array}$ & $\begin{array}{l}\text { 12- HETE } \\
15-\mathrm{HETE}=12-\mathrm{HETE}\end{array}$ \\
\hline rénine & RI & $\begin{array}{l}\text { glomérules } \\
\text { cellules mésangiales }\end{array}$ & $\begin{array}{l}\text { rat } \\
\text { homme } \\
\text { homme }\end{array}$ & prorénine > rénine \\
\hline $\begin{array}{l}\text { activateur tissulaire } \\
\text { du plasminogène }\end{array}$ & $\begin{array}{l}\text { dosage } \\
\text { radioenzymatique }\end{array}$ & glomérules & $\begin{array}{l}\text { homme } \\
\text { rat }\end{array}$ & \\
\hline urokinase & dosage chimique & cellules épithéliales & homme & \\
\hline histamine & dosage chimique & glomérules & rat & \\
\hline dopamine & $\begin{array}{l}\text { dosage radio- } \\
\text { enzymatique }\end{array}$ & glomérules & rat & $\begin{array}{l}\text { taux de synthèse très bas par } \\
\text { rapport au tubule }\end{array}$ \\
\hline $\begin{array}{l}\text { formes actives de } \\
\text { l'oxygène }\end{array}$ & dosage chimique & $\begin{array}{l}\text { glomérules } \\
\text { cellules mésangiales }\end{array}$ & $\begin{array}{l}\text { rat } \\
\text { rat }\end{array}$ & $\mathrm{H}_{2} \mathrm{O}_{2}>\mathrm{O}_{2}$ \\
\hline
\end{tabular}

RI : radioimmunologie; HPLC : chromatographie liquide à haute performance; HETE : acide hydroxyeicosatétraenoïque; $T X$ : thromboxane; $P G$ : prostaglandine.

Les médiateurs les plus étudiés sont les produits de conversion de l'acide arachidonique. Les PG ont été mesurées dans le milieu d'incubation des glomérules murins et humains, soit directement par dosage radioimmunologique, soit après purification par chromatographie liquide à haute performance (HPLC) des dérivés radioactifs provenant de la conversion de l'acide arachidonique radiomarqué par ${ }^{3} \mathrm{H}$ ou ${ }^{14} \mathrm{C}$. Les glomérules de rat synthétisent de manière prédominante la $\mathrm{PGE}_{2}$ et la $\mathrm{PGF}_{2 \alpha}$ alors que les glomérules humains produisent essentiellement la $\mathrm{PGI}_{2}$ et le $\mathrm{TXA}_{2}$ reconnus par leurs métabolites stables, la 6-céto-PGF ${ }_{1 \text { a }}$ et le $\mathrm{TXB}_{2}$ [16]. L'utilisation des cellules glomérulaires en culture a permis de préciser quels types cellu- laires intervenaient dans la synthèse des PG. Les cellules mésangiales de rat produisent environ vingt fois plus de $\mathrm{PGE}_{2}$ et quatre fois plus de $\mathrm{PGF}_{2 \alpha}$ que les cellules épithéliales. Les cellules mésangiales et épithéliales humaines ont une très petite capacité de synthèse des $P G$, due à la faible concentration de cyclooxygénase dans ces cellules. La synthèse de cyclo-oxygènase et, en conséquence, celle de PG peut être restaurée en incubant ces cellules en présence de butyrate qui rend possible l'expression du gène [13]. Les PG jouent un double rôle comme agent vasomoteur et comme médiateur de l'inflammation. Leur synthèse est stimulée par les hormones vasoactives, AII et AVP, mais aussi par des agents de l'inflammation, comme les formes actives de l'oxy- gène, le platelet-activating-factor (PAF) et également, au cours de la phagocytose de particules étrangères par les cellules mésangiales. La stimulation de la synthèse des PG vasodilatatrices va limiter les effets de l'AII, de l'AVP et du PAF sur les fonctions glomérulaires. Ceci peut non seulement jouer un rôle dans la régulation physiologique de la filtration glomérulaire mais aussi dans le développement des glomérulonéphrites. On sait par exemple qu'en plus de son action vasoconstrictrice, l'AII accroît le dépôt des macromolécules dans les glomérules. Un rôle identique a été proposé pour le PAF. La $\mathrm{PGE}_{2}$ et la $\mathrm{PGI}_{2}$ protègent probablement le glomérule de ces effets nocifs, ce qui explique les conséquences néfastes de l'utilisation des inhibiteurs de la 
cyclo-oxygênase sur la fonction rénale et l'action bénéfique de la $\mathrm{PGE}_{2}$ au cours de certains modèles de glomérulonéphrites. Contrairement à la $\mathrm{PGE}_{2}$ et à la $\mathrm{PGI}_{2}$, le $\mathrm{TXA}_{2}$ est un médiateur toxique au cours des glomérulonéphrites. En effet, le traitement par les inhibiteurs de la thromboxane synthétase améliore considérablement le DFG et le flux plasmatique glomérulaire au cours de la néphrite par injection d'anticorps antimembrane basale glomérulaire et également diminue la protéinurie au cours de la glomérulonéphrite induite par l'adriamycine. Il est remarquable que dans ces deux modèles on ait pu montrer que l'excès de $\mathrm{TXA}_{2}$ provenait des cellules glomérulaires elles-mêmes et non des cellules du sang [17].

Le métabolisme de l'acide arachidonique peut se faire également par la voie de la lipoxygénase qui donne naissance à d'autres composés, acides hydroperoxy - eicosatétraénoïques (HPETE), acides hydroxy-eicosatétraénoïques (HETE) et leucotriènes (LT). Les glomérules de rat synthétisent essentiellement le I 2-HETE alors que les glomérules humains synthétisent à la fois le I2et le I 5-HETE [ 18 ]. Le rôle de ces composés dans l'inflammation commence à être analysé. Il a été montré récemment que les produits de la lipoxygénase et, plus particulièrement, le i 2- et le i 5-HPETE étaient responsables de l'adhésion des macrophages aux glomérules in vitro, et de la stimulation des activités de synthèse des $P G$ par ces macrophages [19]. La synthèse de LT par les glomérules est plus incertaine. Les quantités produites sont faibles ce qui ne facilite pas l'identification et laisse dans le doute l'origine, proprement rénale ou à partir des cellules sanguines, de ces composés. En revanche, la synthèse de PAF par les glomérules isolés et les cellules mésangiales paraît assurée. Le PAF est formé à partir du 2-lyso PAF par acétylation en présence d'acétyl transférase. Le PAF stimule la contraction des cellules mésangiales et altère aussi la perméabilité des capillaires glomérulaires en produisant la perte de leurs charges anioniques [20].

Lorsqu'ils sont stimulés, les glomérules et les cellules mésangiales glo- mérulaires synthétisent des formes actives de l'oxygène. Ces substances sont hautement toxiques et provoquent en particulier la dégradation du collagène de la membrane basale glomérulaire et la peroxydation des lipides membranaires. La phagocytose de particules opsonisées par les cellules mésangiales s'accompagne d'une explosion oxydative, avec libération dans le milieu de peroxyde d'hydrogène $\left(\mathrm{H}_{2} \mathrm{O}_{2}\right)$ et d'anion superoxyde $\left(\mathrm{O}_{2}^{-}\right)$[2I]. La synthèse de ces composés dépend du métabolisme de l'acide arachidonique selon des mécanismes encore imparfaitement élucidés. Une première hypothèse fait jouer le rôle principal aux produits issus de l'activité de la phospholipase C, en particulier le diacylglycérol qui stimule la protéine kinase $\mathrm{C}$. Il en résulte l'activation de la NADPH (nicotinamide - adénine - dinucléotide - phosphate) oxydase et la production de formes actives de l'oxygène. Dans une deuxième hypothèse, l'activité de la NADPH oxydase est stimulée par le changement dans la fluidité membranaire, dépendant des acides gras libres. Enfin, l'acide arachido- nique libéré peut être transformé par la lipoxygénase en métabolites, les HPETE et le $\mathrm{LTB}_{4}$ qui, directement ou indirectement, favorisent la production de formes actives de l'oxygène (figure 2). A l'opposé, la transformation de l'acide arachidonique en PG par la voie de la cyclooxygénase limite cette production, probablement par l'intermédiaire de l'augmentation de l'AMP cyclique. La synthèse des radicaux oxygène est également inhibée in vitro par les glucocorticoïdes. Après interaction avec leurs récepteurs, ces hormones stimulent la synthèse d'une protéine spécifique qui inhibe directement ou indirectement la formation des dérivés actifs de l'oxygène. Le rôle des cellules mésangiales glomérulaires résidantes dans la production des médiateurs de l'inflammation est maintenant bien établi. Les stimuli de ces fonctions de synthèse des cellules mésangiales sont la phagocytose de composés immuns, circulants ou déposés dans les glomérules, tels les complexes immuns ou le simple contact avec ces substances. Les composants du complément et le PAF constituent

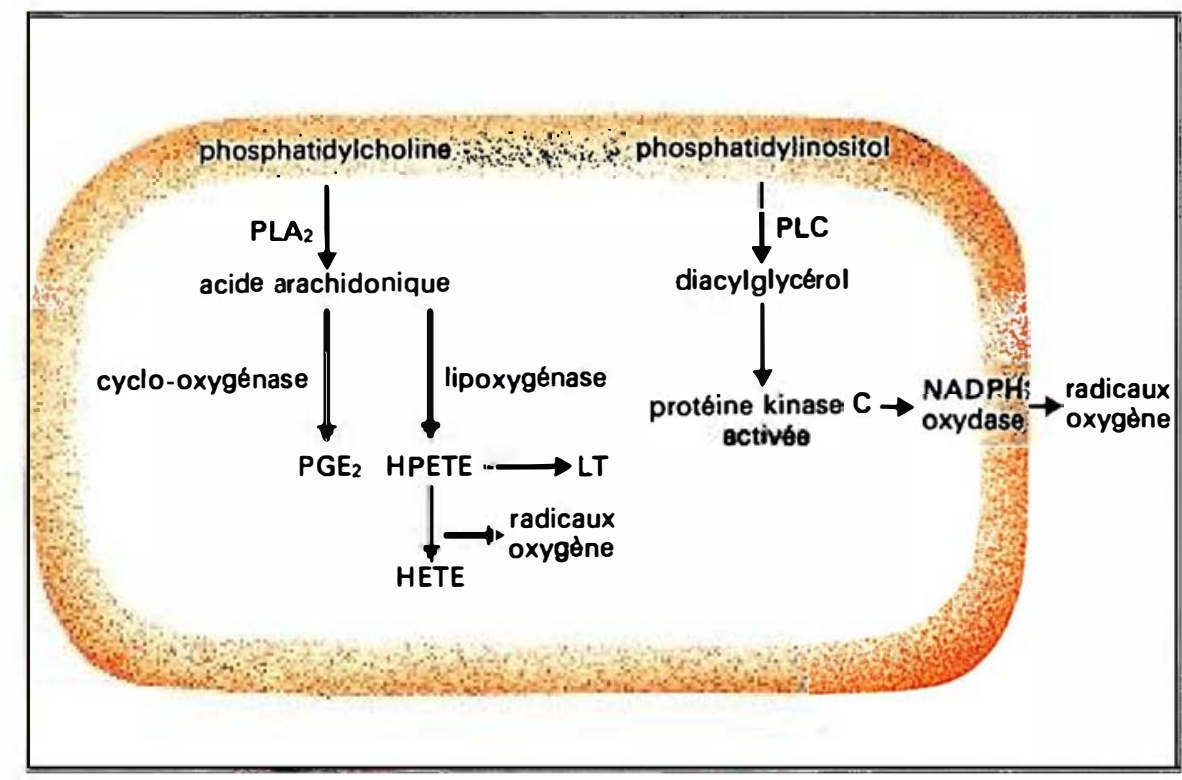

Figure 2. Production des formes actives de l'oxygène en relation avec l'activation des phospholipases membranaires. $P L A_{2}=$ phospholipase $A_{2} ; P L C=$ phospholipase $C$; HPETE = acide hydroperoxy-eicosatétraénoïque; HETE = acide hydroxy-eicosatétraénoïque; $L T=$ leucotriène. 
d'autres stimuli possibles. En plus des PG et des formes actives de l'oxygène, les cellules mésangiales synthétisent des protéases acides et neutres et une cytokine de type interleukine I. De plus, l'identification d'une sous-population de cellules mésangiales porteuses de l'antigène I a et qui donc peuvent présenter des antigènes étrangers aux cellules $T$, suggère que des médiateurs de l'immunité cellulaire peuvent également agir dans le mésangium [22]. Il est tout à fait vraisemblable que ces fonctions des cellules mésangiales puissent jouer un rôle dans le mécanisme des lésions glomérulaires au cours des glomérulonéphrites.

\section{Rénine et érythropoiétine}

La présence de rénine dans les glomérules de rat est bien établie. Les glomérules de rat "superfusés ", c'est-à-dire suspendus dans un milieu d'incubation qui se renouvelle, ont été largement utilisés pour étudier la libération de rénine en conditions basales et en réponse à différents stimuli comme les prostaglandines et les agonistes $\beta$ adrénergiques. Cependant, le lieu précis de la production de rénine dans ces études est encore incertain. Une partie des glomérules isolés retient la portion la plus distale des artérioles afférentes qui peut être la seule source de rénine dans la préparation. Il est également possible que les cellules glomérulaires ellesmêmes possèdent la capacité de produire de la rénine. Contre cette hypothèse, on peut avancer le fait que les études utilisant les techniques d'immunofluorescence, avec un anticorps antirénine humaine, n'ont pas décelé de cellules contenant de la rénine dans les glomérules humains [23]. En fait, la sensibilité de ces techniques constitue une limite sérieuse qui ne permet pas de conclure. Plus récemment, il a été possible de montrer que les cellules mésangiales humaines en culture synthétisaient de la rénine et son précurseur, la prorénine. La rénine a été identifiée avec certitude selon plusieurs critères : activité enzymatique maximum à $\mathrm{pH}$ acide, inhibition de l'activité par un anticorps polyclonal spécifique antirénine et par un inhibiteur pharmacologique de la rénine contenant de la statine [24]. La synthèse de rénine par les cellules mésangiales humaines est stimulée par les prostaglandines et l'isoproterenol. Ainsi les glomérules, dont on sait par ailleurs qu'ils produisent l'enzyme de conversion, sont-ils capables de synthétiser l'angiotensine II, hormone responsable de la vasoconstriction glomérulaire en réponse à l'afflux de sodium dans le tube distal. On a aussi montré récemment que les cellules mésangiales sécrétaient de l'érythropoiétine en réponse à l'anoxie. La synthèse de l'érythropoiétine semble passer par l'intermédiaire des prostaglandines et par la stimulation consćcutive de l'activité adénylate cyclasique [25]. Il s'agit là en fait de la seule hormone (au sens réel du terme) sécrétée par les glomérules puisque l'érythropoiétine agit sur les cellules de la moelle osseuse après avoir été véhiculée par le sang, alors que les autres médiateurs d'origine glomérulaire ont une action essentiellement locale.

\section{Conclusions}

Il ressort deux constatations principales de l'ensemble des travaux sur les récepteurs et les fonctions de synthèse du glomérule. En premier, la régulation endocrinienne du débit de filtration glomérulaire apparaît comme un fait bien établi. L'AII et les $P G$ vasodilatatrices, $\left(\mathrm{PGE}_{2}\right.$ et surtout, $\mathrm{PGI}_{2}$ chez l'homme) occupent une place essentielle dans cette régulation. Deuxième conclusion : les cellules glomérulaires ellesmêmes synthétisent de nombreux facteurs à action inflammatoire locale directe ou indirecte, comme médiateurs, dans ce dernier cas, des fonctions des cellules non résidantes provenant du sang. Le glomérule ne peut donc plus être considéré comme un filtre passif mais comme une structure soumise à la régulation hormonale et surtout pourvue de mécanismes d'autorégulation. Une meilleure analyse de ces mécanismes et de la réponse glomérulaire à l'agression immunologique, bactérienne, toxique ou autre conditionne les progrès des connaissances sur les glomérulonéphrites

\section{Summary}

In vitro studies using isolated glomeruli and glomerular cultured cells of murine and human origin have given a new insight into the glomerular functions and their endocrine control. Glomeruli possess specific receptors for various hormones. The glomerular receptors for angiotensin II exhibit a high affinity, are sensitive to calcium and magnesium concentration and are localized in the mesangial cells. Their number depends on the sodium content of the diet. These receptors are related to two glomerular functions: the contractility of the mesangial cells and the synthesis by these cells of prostaglandins. Other hormones also act on the glomerular functions such as parathyroid hormone via activation of adenylate cyclase and atriopeptides via activation of the particulate guanylate cyclase. Glomeruli convert arachidonic acid into prostaglandins, $\mathrm{PGE}_{2}$ and $\mathrm{PGF}_{2}$ in rats, $\mathrm{PGI}_{2}$ in humans. $\mathrm{PGE}_{2}$ and $\mathrm{PGI}_{2}$ inhibit the vasoconstrictor effects of angiotensin II. Glomeruli also synthesize lipoxygenase products such as i $2-$ and I 5-HETEs. The glomerular lipoxygenase activity mediates in part the attachment of macrophages to glomeruli and the oxidative burst associated with the phagocytosis of particles by mesangial cells. Glomerular mesangial cells also synthesize renin and erythropoietin. The glomerular receptors for hormones and mediators and the glomerular synthetic functions are involved in the regulation of glomerular filtration rate and in the response of the glomeruli to the immunological, infectious or toxic injury.

\section{TIRES A PART}

R. Ardaillou : Inscrm 64, Hôpital Tenon, 4, ruc de la Chinc, 75020 Paris. 